\title{
A practice change intervention to improve antenatal care addressing alcohol consumption by women during pregnancy: research protocol for a randomised stepped-wedge cluster trial
}

Melanie Kingsland ${ }^{1,2,3^{*}}$, Emma Doherty ${ }^{1,2,3}$, Amy E. Anderson ${ }^{2,3}$, Kristy Crooks ${ }^{1,4}$, Belinda Tully ${ }^{1}$, Danika Tremain ${ }^{1,2,3}$, Tracey W. Tsang ${ }^{5,6}$, John Attia ${ }^{2,3}$, Luke Wolfenden 1,2,3, Adrian J. Dunlop ${ }^{2,3,7}$, Nicole Bennett ${ }^{8}$, Mandy Hunter ${ }^{8}$, Sarah Ward ${ }^{9}$, Penny Reeves ${ }^{2,3}$, Ian Symonds ${ }^{10}$, Chris Rissel $^{5,11}$, Carol Azzopardi ${ }^{8}$, Andrew Searles ${ }^{2,3}$, Karen Gillham', Elizabeth J. Elliott ${ }^{5,6}$ and John Wiggers ${ }^{1,2,3}$

\begin{abstract}
Background: Despite clinical guideline recommendations, implementation of antenatal care addressing alcohol consumption by pregnant women is limited. Implementation strategies addressing barriers to such care may be effective in increasing care provision. The aim of this study is to examine the effectiveness, cost and cost-effectiveness of a multi-strategy practice change intervention in increasing antenatal care addressing the consumption of alcohol by pregnant women.
\end{abstract}

Methods: The study will be a randomised, stepped-wedge controlled trial conducted in three sectors in a health district in New South Wales, Australia. Stepped implementation of a practice change intervention will be delivered to sectors in a random order to support the introduction of a model of care for addressing alcohol consumption by pregnant women. A staged process was undertaken to develop the implementation strategies, which comprise of: leadership support, local clinical practice guidelines, electronic prompts and reminders, opinion leaders, academic detailing (audit and feedback), educational meetings and educational materials, and performance monitoring. Repeated cross-sectional outcome data will be gathered weekly across all sectors for the study duration. The primary outcome measures are the proportion of antenatal appointments at 'booking in', 27-28 weeks gestation and 35-36 weeks gestation for which women report (1) being assessed for alcohol consumption, (2) being provided with brief advice related to alcohol consumption during pregnancy, (3) receiving relevant care for addressing alcohol consumption during pregnancy, and (4) being assessed for alcohol consumption and receiving relevant care. Data on resources expended during intervention development and implementation will be collected. The proportion of women who report consuming alcohol since knowing they were pregnant will be measured as a secondary outcome.

* Correspondence: melanie.kingsland@hnehealth.nsw.gov.au

${ }^{1}$ Hunter New England Population Health, Hunter New England Local Health

District, Wallsend, New South Wales, Australia

${ }^{2}$ School of Medicine and Public Health, The University of Newcastle,

Callaghan, New South Wales, Australia

Full list of author information is available at the end of the article

(c) The Author(s). 2018 Open Access This article is distributed under the terms of the Creative Commons Attribution 4.0 International License (http://creativecommons.org/licenses/by/4.0/), which permits unrestricted use, distribution, and reproduction in any medium, provided you give appropriate credit to the original author(s) and the source, provide a link to the Creative Commons license, and indicate if changes were made. The Creative Commons Public Domain Dedication waiver (http://creativecommons.org/publicdomain/zero/1.0/) applies to the data made available in this article, unless otherwise stated. 
(Continued from previous page)

Discussion: This will be the first randomised controlled trial to evaluate the effectiveness, cost and cost-effectiveness of implementation strategies in improving antenatal care that addresses alcohol consumption by pregnant women. If positive changes in clinical practice are found, this evidence will support health service adoption of implementation strategies to support improved antenatal care for this recognised risk to the health and wellbeing of the mother and child.

Trial registrations: Australian and New Zealand Clinical Trials Registry, No. ACTRN12617000882325 (date registered: 16/06/2017).

Keywords: Maternal, Alcohol consumption, Pregnancy, Antenatal care, Implementation, Clinical practice change, Stepped-wedge trial, Protocol

\section{Background}

Maternal alcohol consumption during pregnancy is associated with a number of adverse obstetric, fetal and child outcomes with lifelong consequences. These include, Fetal Alcohol Spectrum Disorder (FASD), miscarriage, stillbirth, preterm birth, congenital anomalies, and low birth weight [1]. No safe level of prenatal alcohol exposure has been established for the foetus and therefore many countries, including Australia, have national guidelines recommending that the safest option is for women to abstain from alcohol consumption when trying to conceive, during conception and during pregnancy [1-3].

Despite this recommendation, $10 \%$ of women worldwide consume alcohol during pregnancy, with notably higher prevalence estimates in regions with high levels of general alcohol consumption, such as the World Health Organisation European Region (prevalence estimate of alcohol consumption in pregnancy, 25\%) [4]. In Australia, national surveys and prospective cohort studies report the prevalence of maternal alcohol consumption at any time during pregnancy to be between 35 and 72\% [5-10]. For example, a prospective cohort study of 1403 women attending antenatal clinics in the Australian states of New South Wales and Western Australia found that $61 \%$ of women consumed alcohol between conception and pregnancy recognition, often at risky levels. Of these women, approximately 30\% continued to drink alcohol once they were aware they were pregnant [10]. Similarly, a study of 1570 women attending public antenatal clinics in Melbourne, Australia, found that just over half (54\%) of women consumed alcohol in the first trimester, and half of these women continued to consume alcohol throughout the remainder of their pregnancy [8].

Health services providing antenatal care represent an opportune setting to address maternal alcohol consumption during pregnancy. Systematic review evidence shows that psychological and educational interventions for pregnant women may reduce alcohol consumption and increase abstinence from alcohol [11]. Additional evidence suggests that clinician assessment of alcohol consumption and brief interventions, including motivational interviewing, may also reduce the risk of an alcohol-exposed pregnancy [12-18]. Efforts to reduce alcohol consumption during pregnancy in antenatal settings is also acceptable to women, with $97 \%$ of Australian women indicating that they wanted information about alcohol use during pregnancy and would be willing to change their alcohol consumption if advised to do so [19]. Consistent with this evidence, international, [20] as well as Australian national [21, 22] and state clinical guidelines $[23,24]$ recommend that health professionals providing antenatal care use a validated tool to assess alcohol consumption for all pregnant women at the initial visit and throughout the antenatal period, provide brief advice about the potential harms of alcohol consumption during pregnancy and recommend abstinence, and refer women to specialist services if they require assistance to stop consuming alcohol.

Despite clear recommendations in clinical guidelines, implementation of antenatal care addressing maternal alcohol consumption during pregnancy is limited. Internationally, studies show that pregnant women are not routinely assessed for their alcohol consumption during antenatal consultations are not consistently provided with information regarding the effects of alcohol consumption during pregnancy and receive inconsistent advice regarding alcohol intake [25-27]. For instance, in Canada, only 50\% of health professionals report providing advice to pregnant women regarding the consumption of alcohol [28] and, in the UK, only two thirds of women reported receiving such advice from a midwife [29]. In Australia, a study of 1143 of health professionals who provide antenatal care found that fewer than half (45\%) routinely asked about alcohol consumption during pregnancy, only $25 \%$ provided information on the effects of alcohol consumption during pregnancy, and only $13 \%$ provided advice consistent with national drinking guidelines [30, 31]. A more recent study involving 166 midwives in Western Australia found that while almost all midwives (93\%) asked pregnant women about their alcohol consumption, only 54\% used a standardised assessment tool [32].

A small number of studies have been conducted to assess barriers to the provision of care addressing maternal alcohol 
consumption during pregnancy. These barriers include a lack of systems and/or tools to prompt clinician assessment of alcohol consumption, concerns about patient sensitivity and stigmatisation, lack of staff time, need for staff training, limited access to or knowledge of clinical and patient resources, including culturally appropriate resources for Aboriginal and Torres Strait Islander women, lack of referral options, a perceived lack of skill in delivering care, and a lack of understanding of the importance of providing such care to all women [33-36]. Additionally, the literature on clinical guideline implementation more broadly indicates that other barriers (including commitment to change from organisational leaders/champions, perceived value/need and readiness to change, skills, ability and confidence, and an absence of systems and tools to support/prompt care delivery) commonly impede changes in professional practice [37].

Cochrane reviews of strategies to improve the implementation of recommended clinical practices suggest that a variety of both organisational and individually focused strategies may be effective. These strategies include leadership, local clinical practice guidelines, electronic prompt and reminder systems, local opinion leaders, educational meetings and educational materials, academic detailing, including audit and feedback, and, monitoring the performance of the delivery of healthcare [38-41]. The effectiveness of such strategies in improving the implementation of guideline recommendations is, however, highly variable [38-42] and, to maximise effectiveness, it is recommended that strategies are selected that target specific barriers to the implementation of recommended clinical practices [43-45]. Implementation frameworks such as the Theoretical Domains Framework have been developed to aid the selection of targeted evidence-based implementation strategies $[46,47]$.

No controlled trials have been conducted to test the effectiveness, cost and cost-effectiveness of implementation strategies in increasing the provision of recommended antenatal care that addresses maternal alcohol consumption during pregnancy. Accordingly, the aim of this study is to examine the effectiveness, cost and cost-effectiveness of a multi-strategy practice change intervention in increasing maternity clinician provision of care addressing the consumption of alcohol by women during their pregnancy.

\section{Methods}

\section{Study design and setting}

The study will be a randomised stepped-wedge controlled trial design conducted in three sectors (clusters) in the Hunter New England Local Health District in New South Wales, Australia. The sectors are geographically defined groupings of antenatal facilities with common operational management. As shown in Fig. 1, repeated cross-sectional outcome data will be gathered on a weekly basis across all three sectors for the duration of the study (34 months). Baseline (current practice/control phase) data will be collected for each of the three sectors from 7 months prior to the commencement of the intervention in the first sector to the start of the intervention in each sector. Stepped implementation of a 7-month practice change intervention will be delivered in a randomly selected order at six monthly intervals. Follow-up data will continue to be collected for all three sectors 7 months following completion of the practice change intervention in the third sector. The outcomes of the trial will be determined by comparing practice change outcomes between the baseline and follow up periods for the three sectors combined.

A randomised stepped-wedge controlled trial design is recommended for the evaluation of complex practice change interventions in settings such as health services as it provides a number of pragmatic and scientific advantages over a randomised controlled trial design $[48,49]$. First, it provides a similar level of evidence as a standard parallel cluster randomised controlled trial (RCT) [50]. Second, although all participants will receive the intervention, its sequential implementation across three sectors provides the capacity to identify secular trends, i.e. changes over time before the intervention is implemented [48]. Third, the design addresses the practical difficulty of recruiting the number of similar antenatal services that would be required for a parallel cluster RCT, instead allowing each cluster to act as its own control $[48,49]$. Finally, the design provides an opportunity for all participating services and women to receive the intervention, overcoming ethical and logistical challenges arising from withholding the intervention [50].

Public antenatal services are the largest provider of antenatal health care in Australia, providing services to a diverse population [51]. The antenatal services in the three sectors service urban and rural areas and provide care to over 6000 women annually, accounting for approximately $70 \%$ of births in Hunter New England Local Health District public hospitals [52].

\section{Random allocation and blinding}

A statistician who is independent of intervention development and implementation will randomly allocate the order in which the intervention is implemented (stepped) across the three health sectors. The random sequence will be generated using a computerised random number generator with allocation undertaken for all three sectors at the one time. Study personnel involved in collecting outcome data will be blind to the allocated order of the delivery of the intervention across the sectors. Participants providing outcome data will not be informed of the experimental nature of intervention implementation across services and therefore will be blind to the stage of study occurring in the service they attended. Given the practice change 


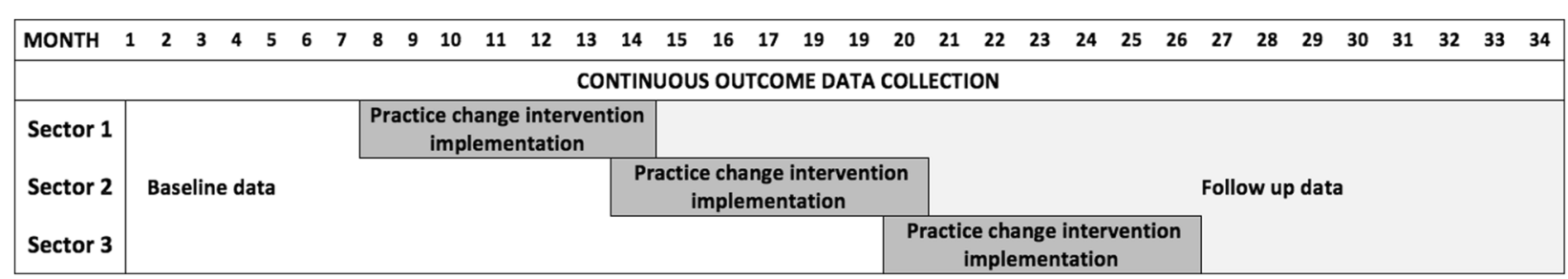

Fig. 1 Study design. Figure 1 shows the trial design and implementation of the trial data collection and intervention components over the course of the 34 months trial period. Repeated cross-sectional outcome data from surveys of pregnant women will be gathered on a weekly basis across all three sectors for the duration of the study. Baseline data will be collected for each of the three sectors from 7 months prior to the commencement of the intervention in the first sector to the start of the intervention in each sector. Stepped implementation of a 7-month practice change intervention will be delivered in a randomly selected order at six monthly intervals. Follow-up data will continue to be collected for all three sectors 7 months following completion of the practice change intervention in the third sector

nature of the intervention, clinicians in antenatal services will be aware when their service is in the intervention period.

\section{Participant eligibility and recruitment Antenatal services and clinicians}

All public antenatal services in the three sectors will receive the practice change intervention, including midwifery group practices, midwifery clinics, specialist medical services, Aboriginal Maternal Infant Health Services (AMIHS), and multi-disciplinary teams caring for women with complex pregnancies or identified vulnerabilities. The practice change intervention will be provided to all maternity clinicians providing antenatal care in participating services: registered midwives (clinical midwife educators, clinical midwife specialists, clinical midwife consultants, community liaison midwives), medical practitioners (staff specialists in obstetrics, fellows, registrars, resident medical officers, general practice obstetricians), Aboriginal health practitioners, Aboriginal health workers and students. All such clinicians who worked in participating antenatal services for at least one of the 7 months during which the practice change intervention was implemented in their sector will be invited to participate in a post intervention survey.

\section{Pregnant women}

It is intended that all pregnant women who attend participating services from the start of the practice change intervention in their health sector will receive the intervention. During the 34-month data collection period, women who attend an individual face-to-face antenatal appointment at three time points (i) the time of the first public antenatal service visit (from this point referred to as the 'booking in' visit), (ii) 27-28 weeks gestation, or (iii) 35-36 weeks gestation, will be eligible to participate in data collection surveys. To be eligible for participation in such surveys, women need to: be aged 18 years or older, be currently pregnant at more than 12 weeks gestation and less than 37 weeks gestation, have a sufficient level of English language proficiency to complete the survey unaided, and be mentally and physically capable of completing the survey. Women will be ineligible to participate in data collection surveys if they: are determined by clinical discretion to be inappropriate to contact for the survey (e.g. due to medical or social issues); and/or are receiving the majority of their antenatal care via a private obstetrician; and/or have given birth or had a negative pregnancy outcome; and/or had already been selected to participate in the survey for that care time point in the past 4 weeks; and/or had previously declined participation in the survey. The number of women deemed ineligible for the above-listed reasons will be recorded and reported.

Each week, a sample of eligible women who attended an antenatal appointment in the past week (for booking in, 27-28 weeks gestation or 35-36 weeks gestation care) will be randomly selected via a computerised random number generator by members of the research team not involved in delivering care to women. Selected women will be mailed a participant information statement explaining the purpose of the survey 1 week prior to receiving a phone call inviting them to participate in the survey. Study posters will be displayed in antenatal clinics and pamphlets distributed in antenatal information packs provided to all women at the time of their booking in appointment. Women identified via the medical record data as being of Aboriginal or Torres Strait Islander origin and/or women who are attending or enrolled to attend an AMIHS will be first contacted by text message and invited to participate. If they do not respond, they will be followed up with a telephone call 4 days later. All women will have the opportunity to decline participation at any point, including opting out during the clinic visit or when they receive information in the antenatal booking in pack, when they receive the study information letter in the mail, at the time of the phone call or text message, or partway through survey completion. On the morning of the day that a woman is to be contacted via phone call or text message, medical 
record data will be checked and any women who are identified as having given birth or having had a negative pregnancy outcome will be deemed ineligible and not contacted.

\section{Intervention}

Model of care for addressing maternal alcohol consumption in pregnancy

A model of care for addressing alcohol consumption in pregnancy will be implemented in antenatal services across the three participating sectors. The model of care is consistent with international [20] and Australian national $[21,22]$, and state [23, 24] antenatal clinical practice guidelines and is based on models of assessment and brief intervention that have been shown to reduce the risk of an alcohol-exposed pregnancy [11, 13-18].

As shown in Fig. 2, the model of care will consist of three key elements-assessment, advice and referral, which will be delivered to women who attend an antenatal clinic appointment the booking in, 27-29 weeks gestation and 35-37 weeks gestation.

\section{1) Assessment of alcohol consumption}

The Alcohol Use Disorders Identification Test Consumption (AUDIT-C) tool will be used to assess the alcohol consumption of pregnant women. The tool has shown to be a valid tool for use with pregnant women [53]. Maternity clinicians will ask all women the first AUDIT-C question: 'How often do you have a drink containing alcohol?'. Those women who provide a response other than 'Never', will be asked the remaining two AUDIT-C items: 'How many standard drinks of alcohol do you have on a typical day when you are drinking?' and 'How often do you have five or more alcohol drinks on one occasion?'. These responses will be used to calculate an 'Alcohol Risk Score' and 'Alcohol Risk of Harm' category (No Risk, Low Risk, Medium Risk, High Risk).

\section{2) Provision of brief advice}

All women, regardless of their Alcohol Risk of Harm category, will be provided with advice by their maternity clinician that it is best not to consume alcohol at any time during pregnancy and that alcohol consumption during pregnancy can increase risk of harm to the foetus and the woman. Women will be provided with additional advice based on their Alcohol Risk of Harm category.

3) Referral to specialist services for additional support

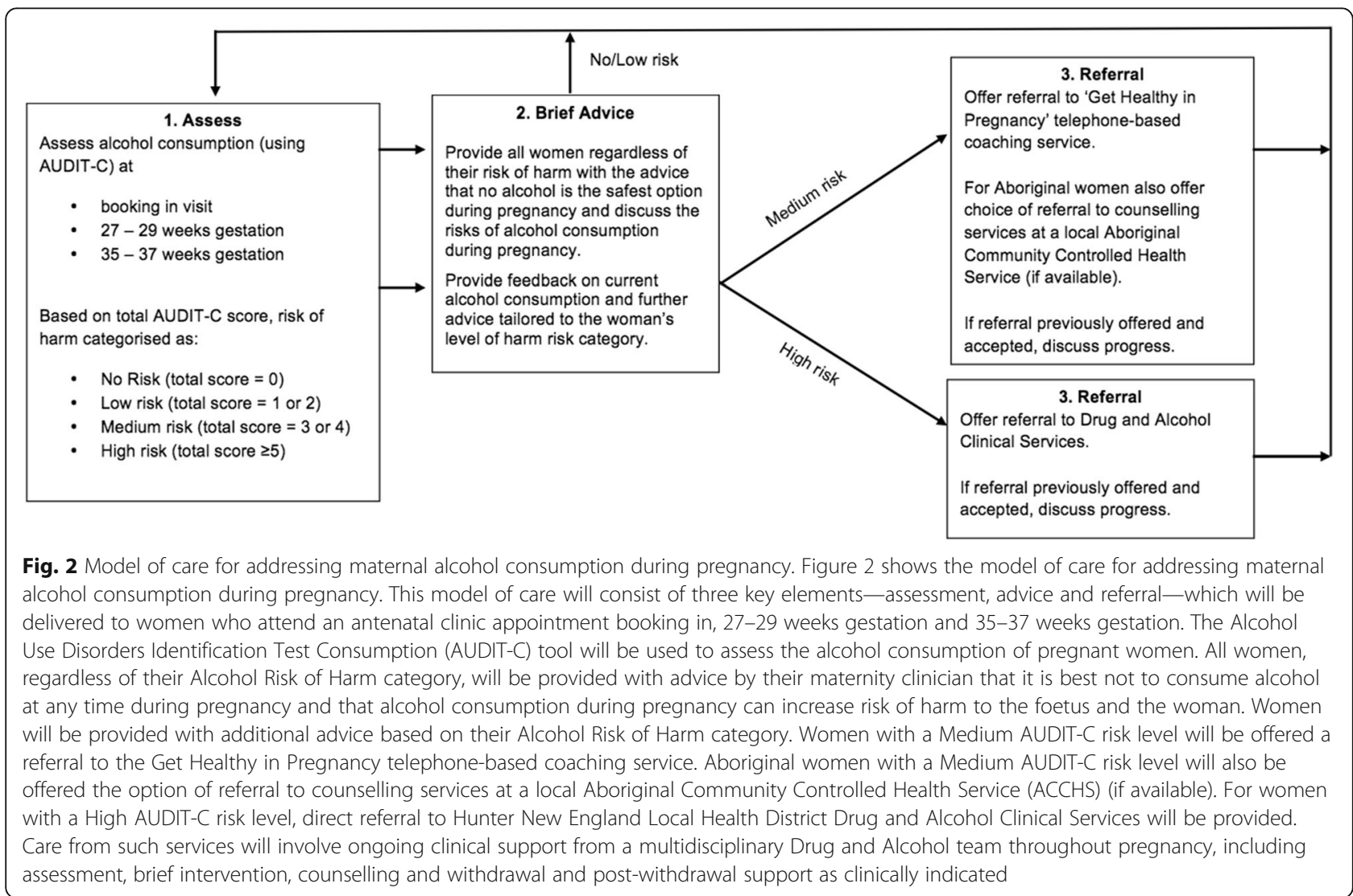


Women with a Medium AUDIT-C risk level will be offered a referral to the Get Healthy in Pregnancy telephone-based coaching service. Get Healthy in Pregnancy is a free, state-wide, government-funded telephone coaching service that supports women to make positive health and lifestyle changes [54]. Women can set a goal of alcohol abstinence throughout pregnancy and receive up to ten tailored calls by qualified health coaches. The coaching is based on behaviour change principles designed to assist with goal setting, maintaining motivation and overcoming barriers [54]. Aboriginal women with a Medium AUDIT-C risk level will also be offered the option of referral to counselling services at a local Aboriginal Community Controlled Health Service (ACCHS) (if available). This latter referral pathway was established through consultation with Aboriginal community members and the ACCHS's that provide care for Aboriginal pregnant women in the study region.

For women with a High AUDIT-C risk level, direct referral to Hunter New England Local Health District Drug and Alcohol Clinical Services will be provided. Care from such services will involve ongoing clinical support from a multidisciplinary Drug and Alcohol team throughout pregnancy, including assessment, brief intervention, counselling and withdrawal and post-withdrawal support as clinically indicated. Referral may also be made to residential drug and alcohol treatment services if required (provided by non-government services).

\section{Implementation intervention}

A series of organisational and individual clinician focused strategies will be used to support clinician implementation of the model of care.

Implementation intervention strategy development The following staged process was undertaken to develop the implementation strategies:

1. Quantitative anonymous surveys were undertaken with 33 clinicians working in antenatal services across the three sectors and an additional eight managers of maternity services/teams to determine perceived barriers and enablers to their implementation of the model of care for addressing maternal alcohol consumption during pregnancy. The surveys were constructed based on 11 domains of the Theoretical Domains Framework (TDF) $[55,56]$ and were informed by previous surveys of health professionals [57] and previous studies utilising the TDF across different health care settings [58-60].

2. The priority barriers identified through the clinician and manager surveys were mapped to TDF techniques for behaviour change [43] and a proposed list of implementation strategies was developed based on a review of the literature and advice sought from experts in treatment of alcohol harms, clinical practice change, health service research and behavioural sciences.

3. Consultation with Aboriginal community members, ACCHSs within the participating sectors, AMIHS staff, and Aboriginal population health staff was undertaken to ensure the content of implementation strategies was culturally appropriate for women. Qualitative research (focus groups) was also conducted with Aboriginal mothers (who had attended a public antenatal service in the study region in the last 12 months) and Aboriginal pregnant women to explore experiences of antenatal care in relation to the consumption of alcohol during pregnancy and identify any issues relating to the provision of such care to ensure the implementation strategies were culturally appropriate.

4. Final refinement of implementation strategies and development of strategy content was undertaken following consultation with key clinicians and managers across the three participating health sectors.

Implementation intervention strategies Table 1 lists the implementation strategies that will be used to support the introduction of the model of care for addressing maternal alcohol consumption during pregnancy (Fig. 2), including the evidence source and the TDF domains on which each strategy was based and the barriers that the strategy was intended to address.

Implementation intervention delivery timeline The intervention strategies listed above will be implemented in each sector for 7 months prior to follow up data collection (Fig. 1). This will include a 1-month period introducing the practice change and a 6-month intensive practice change intervention. Given their organisational and system focus, all strategies, other than the local opinion leader (Clinical Midwife Educator (CME)) and academic detailing strategies, have the potential to continue to be implemented following the 7-month study intervention period, subject to the operational decisions of the Local Health District.

\section{Control and contamination Usual care}

Prior to implementation of the practice change intervention in each of the three sectors, usual antenatal care for addressing maternal alcohol consumption during pregnancy will be provided. Such care is likely to vary by antenatal service and clinician as no existing health sector-wide guideline 


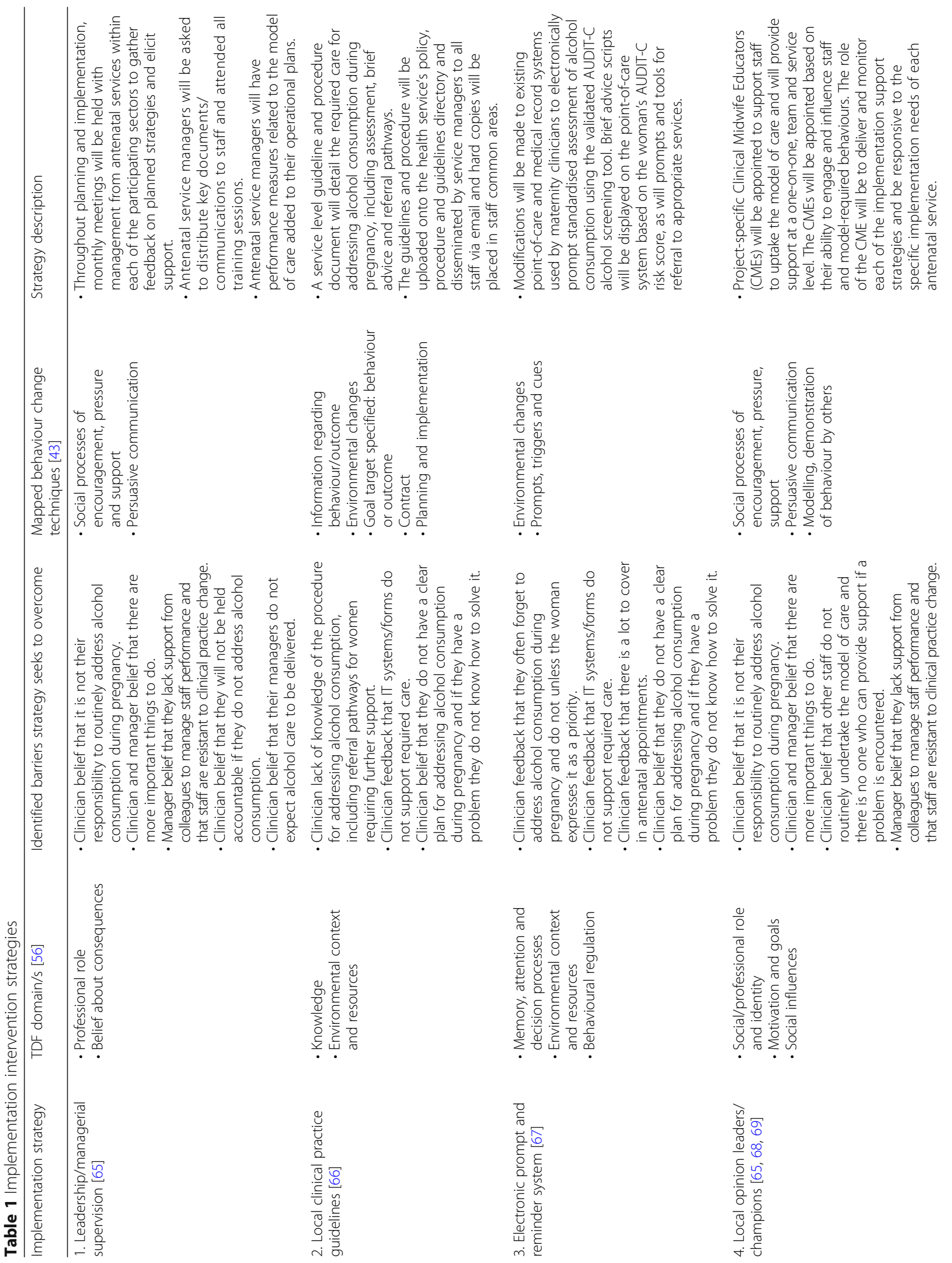




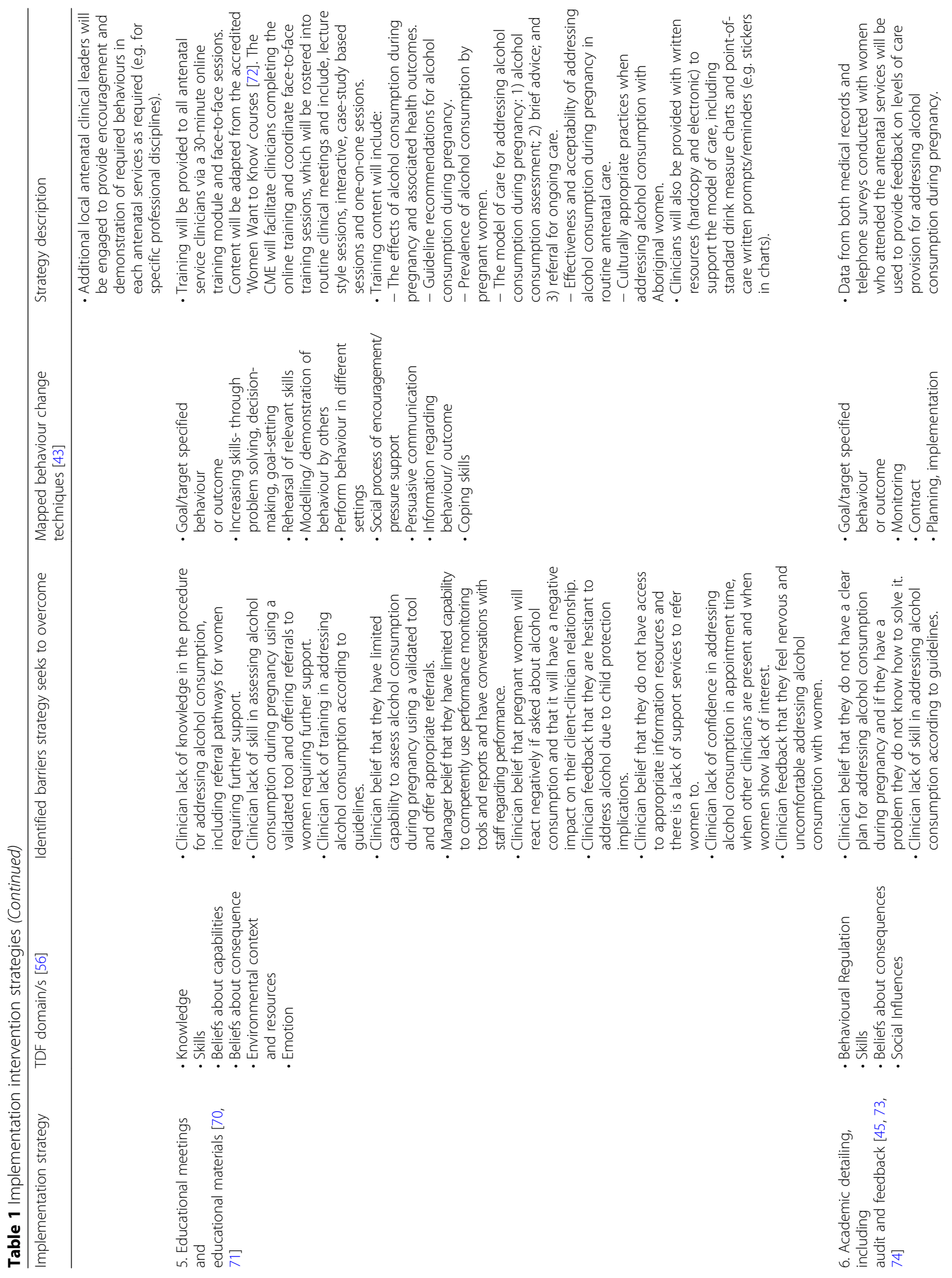




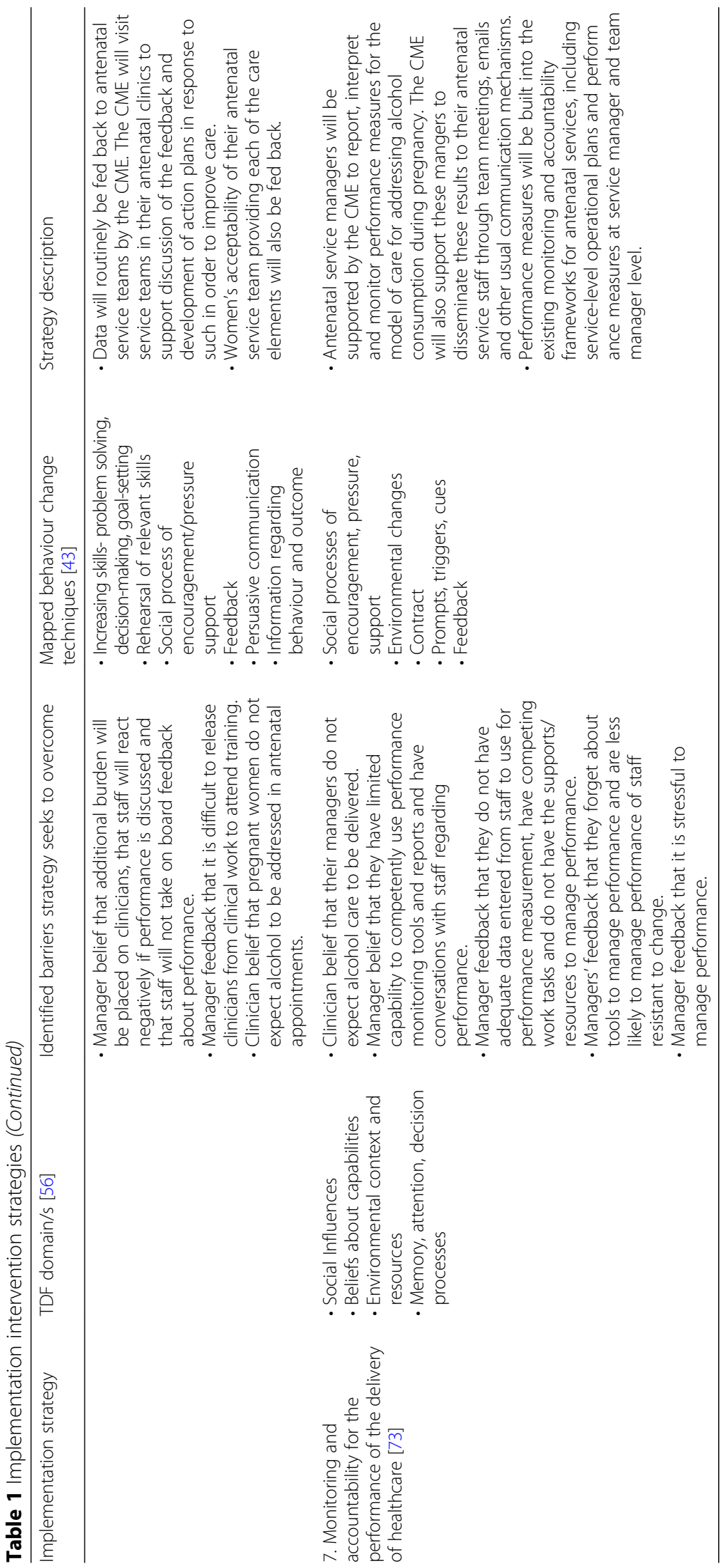


or procedure specifies the provision of routine care for addressing alcohol consumption during pregnancy.

\section{Potential for contamination}

As the research team will control the initiation and delivery of all the intervention elements, the intervention strategies will not be accessible to antenatal clinicians during the baseline (control) phase. Although potential for contamination during this phase from staff movement between sectors is possible, it is considered to be limited due to the structural and systemic nature of the implementation strategies. Information on movement of clinicians between participating sectors will be collected throughout the study.

\section{Measures}

\section{Primary trial outcomes}

There are four primary outcomes for this trial. They are the proportion of all antenatal clinic appointments (at 'booking in', 27-28 weeks gestation and 35-36 weeks gestation) for which women report:

1. Being assessed for alcohol consumption and level of risk using the AUDIT-C.

2. Being provided with brief advice related to alcohol consumption during pregnancy.

3. Receiving, relative to their level of risk, the relevant elements of antenatal care for addressing alcohol consumption during pregnancy (advise and refer).

4. Being assessed for alcohol consumption and level of risk using the AUDIT-C and receiving, relative to their level of risk, the relevant elements of antenatal care for addressing alcohol consumption during pregnancy (advise and refer).

\section{Secondary trial outcome}

For women attending antenatal appointments at 'booking in', 27-28 weeks gestation and 35-36 weeks gestation, alcohol consumption since pregnancy recognition as measured by total AUDIT-C score will be collected based on self-report of women. AUDIT-C is a validated tool for assessing risk of harm due to alcohol consumption [53].

\section{Process evaluation measures}

The acceptability, appropriateness, feasibility, intervention fidelity and reach of the model of care for addressing maternal alcohol consumption in pregnancy and the implementation strategies will be assessed via surveys of women and clinicians and project records. These process measures will be based on an implementation evaluation framework [61] and use validated measures where available [62]. Measures to assess implementation intervention reach will include participation of antenatal clinical staff in educational meetings, interaction with local opinion leaders, involvement in academic detailing/ audit and feedback sessions, and receipts of clinical practice guidelines. To determine reach of the implementation intervention strategies across different groups of clinicians, data will be collected from clinicians on position/ profession, level of training, and length of time working in current antenatal service and in antenatal services generally. To assess delivery of the model of care across different demographic groups of women, the following information will be collected from pregnant women: age, gender, highest level of education, employment status, geographical location, Aboriginal or Torres Strait Islander status of woman and baby, household composition, current gestation, gestation at first antenatal clinic visit, whether attending care for first or subsequent pregnancy and alcohol consumption prior pregnancy (via AUDIT-C).

\section{Cost and cost-effectiveness}

To provide a measure of the investment required to develop, implement and maintain the effect of the implementation strategies, resource use will be prospectively measured and valued from a public finance perspective. The outcomes from the cost analysis will be (i) an estimate of the cost required to develop the intervention strategies, (ii) the net cost of delivering the implementation intervention (including labour costs for the CME and the clinicians to conduct/participate in each of the implementation strategies, undertaking quality assurance processes, providing managerial oversight), and (iii) assuming a positive trial outcome, the expected incremental cost to maintain effect. Additionally, the cost-effectiveness of the implementation strategies will be assessed relative to the baseline (current practice) phase in each of the three sectors. The incremental cost-effectiveness ratio (ICER) will be calculated as the difference in average cost between the intervention and baseline phases, divided by the difference in the primary outcome. Sensitivity analyses will test the robustness of results to selected issues and assumptions.

\section{Data collection procedures Primary and secondary outcome measures}

Each week a sample of women who in the past week attended an antenatal clinic for a 'booking in' visit, a visit when they were $27-28$ weeks gestation, or a visit when they were 35-36 weeks gestation will be sent a letter providing information about the study and inviting them to participate in a computer assisted telephone interview (CATI) survey. Telephone contact will be attempted with women up to ten times over a 2-week period, including at different times of the day and on weekdays and weekends, in order to elicit consent and completion of the survey. If a woman declines to participate in the CATI, they will be invited to complete the survey online. If they consent to participate in the online survey, they 
will be sent a survey link via text message. Women who are of Aboriginal or Torres Strait Islander origin and/or are attending or enrolled to attend an AMIHS will be offered via text message the choice of participating in the survey via either CATI or online mode.

The CATI survey will be conducted by experienced female interviewers who will receive specific training and undertake practice interviews. The CATI and online survey script are identical in the wording of questions, response options and help provided. Both surveys will be pilot tested prior to starting the study to test comprehension, logic flow and survey length.

\section{Process evaluation}

Data for the aforementioned process evaluation measures will be collected via surveys with women (as per procedure described above) and clinicians. Online surveys of clinicians will be conducted at the completion of the intervention in each sector. All eligible clinicians in antenatal services in the participating sectors will be sent a link to an online survey via email, and also given the option to complete the survey on tablet computers/laptops in regular in-services and clinic meetings. Surveys will be completed anonymously. Additional process data to assess intervention fidelity and reach will be collected using project management logs completed by project staff.

\section{Cost and cost effectiveness}

Project management logs, including a cost capture template, will be used to prospectively collect data regarding the resources expended during intervention development and implementation.

\section{Overall data management}

Management of trial data will be in accordance with a data management protocol, which has been developed and approved by the project's advisory group. Data will be stored securely as per the requirements of the Hunter New England Human Research Ethics Committee, The University of Newcastle Human Research Ethics Committee and the Aboriginal Health and Medical Research Council. Data will only be accessible to primary researchers and statisticians. Confidential participant data will be stored securely and not linked to survey responses.

\section{Sample size and power calculations}

It is expected that $70 \%$ of invited women will consent to participate in the surveys (based on previous work by the research team [63]). Assuming 48 working weeks a year, and an intra-class correlation of 0.01 , a sample of 200 women per month (approximately 67 women per each of the three time points: booking in visit, $27-28$ weeks gestation, 35-36 weeks gestation) will give the study $80 \%$ power to detect an absolute increase in care provision of
$15 \%$ in the intervention period (based on a conservative $50 \%$ estimate of prevalence of care provision at baseline) in at least one of the four primary outcomes at a $1.25 \%$ significance threshold. Assuming there are approximately 190 women eligible per week, a weekly sample of 72 women (i.e. 24 women per time point) with a $70 \%$ survey completion rate $(n=50)$ will result in the required number of women needed per month.

\section{Statistical analysis}

Baseline and follow-up primary outcomes data will be analysed using a logistic mixed model to detect change over time in the reported receipt of recommended antenatal care for addressing alcohol consumption during pregnancy. For the secondary trial outcome, linear mixed models will be used to analyse changes in AUDIT-C scores of participants between baselines and follow up periods. The models will have a period term (fixed effect, reflecting pre-post difference, the main indicator of effect) and health sector term (fixed effect). Where appropriate, the models will also include fixed effects for client group (booking in, 2728 weeks gestation, 35-36 weeks gestation) and a time term (fixed effect, to pick up any secular trend). In the latter models, a client group by period term will detect differences between the client groups in their response to the intervention. Where the interaction terms are significant, subgroup analyses will be reported for each of the three client groups. Descriptive statistics will be used to report on process measures and interventions costs. Process outcomes will be used to narratively interpret the results of primary outcome analysis. SAS (V9.3 or later) will be used for all statistical analyses.

\section{Research trial governance}

A research co-production approach has been employed in the development and design of the study [64]. The conduct of the study will similarly be overseen by an advisory group consisting of researchers, policy makers, practitioners and clinical experts with expertise related to alcohol, health promotion, implementation science, FASD, obstetrics and maternal health. A project team consisting of research staff and practitioners will develop and operationalise implementation strategies and data collection components of the trial according to study protocol. Local clinical experts based at each of the three participating sectors will provide advice on aspects of the model of care and implementation strategies that require sector-specific tailoring.

\section{Aboriginal cultural governance}

A series of Aboriginal cultural governance task groups, co-led by Aboriginal and non-Aboriginal staff, will provide guidance on cultural considerations for Aboriginal and Torres Strait Islander people relating to the model of care, 
implementation strategies, data collection, and interpretation and dissemination of study findings.

\section{Trial discontinuation or modification}

There are no criteria for trial discontinuation as it is not anticipated that any events would occur that would warrant discontinuing the trial. Any unforeseen adverse events will be reported to the Hunter New England Human Research Ethics Committee (primary approval committee) and advice sought regarding required action. The trial registration record will be updated with any protocol modifications and any deviations from original protocol will be reported in study outcome papers.

\section{Discussion}

Despite the need, there is a clear absence of research evidence of the effectiveness, cost and cost-effectiveness of implementation strategies to improve antenatal care that addresses maternal alcohol consumption during pregnancy. This will be the first randomised controlled trial to evaluate the effectiveness of such intervention strategies. The stepped-wedge design is feasible and acceptable in the context of conducting a trial across multiple antenatal services. The study has strong design elements including random allocation of the order of strategy implementation across the health sectors and blinding of data collection staff. The implementation intervention strategies have been developed based on key implementation science frameworks and using data from surveys with antenatal services staff and managers. A research co-production approach has been employed in the design of the study and will be employed in its conduct and dissemination.

If positive changes in clinical practice are found, the study will provide evidence to support the delivery by health services of the implementation strategies to improve antenatal care addressing this recognised risk to the health and wellbeing of both the mother and child. The methods used in this trial have the potential to provide a framework for the development of initiatives for improving the implementation of models of care, both in the antenatal clinic setting and in other clinical environments.

\section{Abbreviations \\ ACCHS: Aboriginal Community Controlled Health Service; AMIHS: Aboriginal Maternal Infant Health Service; AUDIT-C: Alcohol Use Disorders Identification Test-Consumption; CATI: Computer-assisted telephone interview; \\ CME: Clinical Midwife Educator; FASD: Fetal alcohol spectrum disorder; \\ ICER: Incremental cost-effectiveness ratio; RCT: Randomised controlled trial; \\ TDF: Theoretical Domains Framework}

\section{Funding}

This protocol is for a research project funded by the National Health and Medical Research Council (NHMRC) Partnership Project grant (APP1113032). The NHMRC has not had any role in the design of the study as outlined in this protocol and will not have a role in data collection, analysis of data, interpretation of data and dissemination of findings. As part of the NHMRC Partnership Grant funding arrangement, the following partner organisations also contribute fund: Hunter New England Local Health District Clinical
Services Nursing and Midwifery, the Foundation for Alcohol Research and Education, and the NSW Health Office of Preventive Health. Individuals in positions that are fully or partly funded by these partner organisations (as described in 'Competing Interests') will have a role in the study design, data collection, analysis of data, interpretation of data and dissemination of findings. The University of Newcastle will make final decisions on each of these study aspects.

\section{Authors' contributions}

MK and JW led the overall development of the research protocol and MK led the development of the manuscript. TS and EE contributed to the development of the rational and background for the protocol. ED, AA, KC, $B T, A D, N B, M H, S W, I S, C R, C A, K G$ and EE contributed to the development of the model of care and implementation support strategies. DT, AA, KC and ED contributed to the development of data collection methods generally and PR and AS contributed to the development of data collection methods specific to the cost and cost-effectiveness measures. LW and JA provided overall guidance for the study design and data analysis. All authors read and approved the final manuscript.

Ethics approval and consent to participate

This study was approved by the Hunter New England Human Research Ethics Committee (16/11/16/4.07; 16/10/19/5.15), the Aboriginal Health and Medical Research Council (1236/16) and the University of Newcastle Human Research Ethics Committee (H-2017-0032; H-2016-0422).

Consent for publication

Not applicable.

\section{Competing interests}

Authors MK, ED, KC, BT, DT, LW, NB, MH, CA, KG and JW receive salary support from Hunter New England Clinical Services Nursing and Midwifery, which contributes funding to the project outlined in this protocol. Similarly, Author CR and Author SW receive salary support from the New South Wales Health Office of Preventive Health and the Foundation for Alcohol Research and Education, respectively. Both of these organisations also contribute funding to this project. All other authors declare that they have no competing interests.

\section{Publisher's Note}

Springer Nature remains neutral with regard to jurisdictional claims in published maps and institutional affiliations.

\section{Author details}

${ }^{1}$ Hunter New England Population Health, Hunter New England Local Health District, Wallsend, New South Wales, Australia. ${ }^{2}$ School of Medicine and Public Health, The University of Newcastle, Callaghan, New South Wales, Australia. ${ }^{3}$ Hunter Medical Research Institute, New Lambton Heights, New South Wales, Australia. ${ }^{4}$ Menzies School of Health Research, Charles Darwin University, Darwin, Northern Territory, Australia. ${ }^{5}$ School of Medicine, The University of Sydney, Camperdown, New South Wales, Australia. ${ }^{6}$ Sydney Children's Hospital Network, Kids' Research Institute, Westmead, New South Wales, Australia. ${ }^{7}$ Drug and Alcohol Clinical Services, Hunter New England Local Health District, Newcastle, New South Wales, Australia. ${ }^{8}$ Maternity and Gynaecology John Hunter Hospital, New Lambton Heights, New South Wales, Australia. ${ }^{9}$ Foundation for Alcohol Research and Education, Deakin, Australian Capital Territory, Australia. ${ }^{10}$ Adelaide Medical School, The University of Adelaide, Adelaide, South Australia, Australia. ${ }^{11} \mathrm{New}$ South Wales Office of Preventive Health, Liverpool, New South Wales, Australia.

Received: 5 July 2018 Accepted: 7 August 2018

Published online: 20 August 2018

References

1. National Health and Medical Research Council (NHMRC). Australian guidelines to reduce health risks from drinking alcohol. Canberra: Commonwealth of Australia; 2009.

2. Butt P, Beirness D, Gliksman L, Paradis C, Stockwell T. Alcohol and health in Canada: a summary of evidence and guidelines for low risk drinking. Ottawa: Canadian Centre on Substance Abuse; 2011. 
3. The Danish National Board of Health. Healthy habits - before, during and after pregnancy. 1st English edition (translated from the 2nd Danish edition). Copenhagen South: The Danish National Board of Health and The Danish Committee for Health Education; 2010.

4. Popova S, Lange S, Probst C, Gmel G, Rehm J. Estimation of national, regional, and global prevalence of alcohol use during pregnancy and fetal alcohol syndrome: a systematic review and meta-analysis. Lancet Glob Health. 2017;5:e290-9.

5. O'Keeffe LM, Kearney PM, McCarthy FP, Khashan AS, Greene RA, North RA, Poston L, McCowan LM, Baker PN, Dekker GA, et al. Prevalence and predictors of alcohol use during pregnancy: findings from international multicentre cohort studies. BMJ Open. 2015;5:e006323.

6. Australian Institute of Health and Welfare. National Drug Strategy Household Survey detailed report 2016. Drug statistics series no 31. Cat. no. PHE 214. Canberra: AlHW; 2017.

7. Cameron CM, Davey TM, Kendall E, Wilson A, McClure RJ. Changes in alcohol consumption in pregnant Australian women between 2007 and 2011. Med J Aust. 2013;199:355-7.

8. Muggli E, O' Leary C, Donath S, Orsini F, Forster D, Anderson PJ, Sharon L, Nagle C, Craig JM, Elliott E, Halliday J. "Did you ever drink more?" A detailed description of pregnant women's drinking patterns. BMC Public Health. 2016;16:683.

9. Anderson AE, Hure AJ, Forder PM, Powers J, Kay-Lambkin FJ, Loxton DJ. Risky drinking patterns are being continued into pregnancy: a prospective cohort study. PLoS One. 2014;9:e86171.

10. McCormack C, Hutchinson D, Burns L, Wilson J, Elliott E, Allsop S, Najman J, Jacobs S, Rossen L, Olsson C, Mattick R. Prenatal alcohol consumption between conception and recognition of pregnancy. Alcohol Clin Exp Res. 2017:41:369-78.

11. Stade BC, Bailey C, Dzendoletas D, Sgro M, Dowswell T, Bennett D. Psychological and/or educational interventions for reducing alcohol consumption in pregnant women and women planning pregnancy. Cochrane Database Syst Rev. 2009;(2):CD004228.

12. Nislen $P$. Brief alcohol intervention to prevent drinking during pregnancy: an overview of research findings. Curr Opin Obstet Gynecol. 2009;21:496-500.

13. Floyd R, Sobell M, Velasquez $M$, et al. Preventing alcohol-exposed pregnancies: a randomized controlled trial. Am J Prev Med. 2007:32:1-10.

14. Floyd R, Weber M, Denny C, et al. Prevention of fetal alcohol spectrum disorders. Dev Disabil Res Rev. 2009;15:193-9.

15. Chang G, McNamara T, Orav E, et al. Brief intervention for prenatal alcohol use: a randomized trial. Obstet Gynecol. 2005;105:991-8.

16. Rendall-Mkosi K, Morojele N, London L, et al. A randomized controlled tria of motivational interviewing to prevent risk for an alcohol exposed pregnancy in the Western Cape, South Africa. Addiction. 2013;108:725-32.

17. Fleming $M$, Lund $M$, Wilton $G$, et al. The healthy moms study: the efficacy of brief alcohol intervention in postpartum women. Alcohol Clin Exp Res. 2008; 32:1600-6.

18. O'Connor M, Whaley S. Brief intervention for alcohol use by pregnant women. Am J Public Health. 2007;97:252-8.

19. Peadon E, Payne J, Henley N, O'Leary C, D'Antoine H, Bartu A, Bower C, Elliot E. How do women want to be informed about alcohol use in pregnancy? In: Book of Abstracts 4th International Conference on Fetal Alcohol Spectrum Disorders 2011; 2011.

20. World Health Organisation. Guidelines for the identification and management of substance use and substance use disorders in pregnancy. Geneva: WHO; 2014

21. The Royal Australian and New Zealand College of Obstetricians and Gynaecologists: Alcohol in pregnancy. RANZCOG; 2014.

22. Australian Health Ministers' Advisory Council. Clinical practice guidelines: antenata care - module I. Canberra: Australian Government Department of Health; 2012.

23. NSW Ministry of Health. Clinical guidelines for the management of substance use during pregnancy, birth and the postnatal period. North Sydney: NSW Ministry of Health; 2014.

24. Queensland Clinical Guidelines. Perinatal substance use: maternal. Brisbane: Queensland Government; 2016.

25. Jones S, Eval M, Talenta J, et al. Midwives and pregnant women talk about alcohol: what advice do we give and what do they receive? Midwifery. 2011;27:489.

26. Jones S, Talenta J. What influences Australian women to not drink alcohol during pregnancy? Aust J Prim Health. 2012;18:68-73.

27. Loxton D, Chojenta C, Anderson A, et al. Acquisition and utilization of information about alcohol use in pregnancy among Australian pregnant women and service providers. J Midwifery Womens Health. 2013;58:523-30.
28. Public Health Agency of Canada. Knowledge and attitudes of health professionals about fetal alcohol syndrome: results of a national survey. Calgary: Health Canada; 2003.

29. Health and Social Care Information Centre. Infant feeding survey. Dundee: Health and Social Care Information Centre; 2007.

30. Payne JM, Elliott E, D'Antoine HA, et al. Health professionals' knowledge, practice and opinions about fetal alcohol syndrome and alcohol consumption in pregnancy. Aust NZ J Public Health. 2005;29:558-64.

31. Elliott E, Payne JM, Haan E, et al. Diagnosis of fetal alcohol syndrome and alcohol use in pregnancy: a survey of paediatricians' knowledge, attitudes and practice. J Paediatr Child Health. 2006;42:698-703.

32. Payne JM, Watkins RE, Jones HM, Reibel T, Mutch R, Wilkins A, Whitlock J, Bower C. Midwives' knowledge, attitudes and practice about alcohol exposure and the risk of fetal alcohol spectrum disorder. BMC Pregnancy Childbirth. 2014:14:377.

33. Diekman S, Floyd R, Decoufle P, et al. A survey of obstetrician-gynecologists on their patients' alcohol use during pregnancy. Obstet Gynecol. 2000;95:756-63.

34. Payne JM, Frances K, Henley N, et al. Paediatricians' knowledge, attitudes and practice following provision of educational resources about prevention of prenatal alcohol exposure and fetal alcohol spectrum disorder. J Paediatr Child Health. 2011:47:704-10.

35. Williams HM, Percival NA, Hewlett NC, Cassady RBJ, Silburn SR. Online scan of FASD prevention and health promotion resources for Aboriginal and Torres Strait Islander communities. Health Promot J Austr. 2018;29:31-8.

36. Mutch R, Wray J, Bower C. Recording a history of alcohol use in pregnancy: an audit of knowledge, attitudes and practice at a child development service. J Popul Ther Clin Pharmacol. 2012;19:e227-33.

37. Geerligs L, Rankin NM, Shepherd HL, Butow P. Hospital-based interventions: a systematic review of staff-reported barriers and facilitators to implementation processes. Implement Sci. 2018;13:36.

38. Freund M, Campbell E, Paul C, et al. Increasing hospital-wide delivery of smoking cessation care for nicotine-dependent in-patients: a multi-strategic intervention trial. Addiction. 2009;104:839-49.

39. Flodgren G, Parmelli E, Doumit G, et al. Local opinion leaders: effects on professional practice and health care outcomes. Cochrane Database Syst Rev. 2011;(8):CD003030.

40. Reeves S, Perrier L, Goldman J, et al. Interprofessional education: effects on professional prac- tice and healthcare outcomes (update). Cochrane Database Syst Rev. 2013;(3):CD002213.

41. Ivers $\mathrm{N}$, Jamtvedt $\mathrm{G}$, Flottorp S. Audit and feedback: effects on professional practice and healthcare outcomes. Cochrane Database Syst Rev. 2012;(6):CD000259.

42. Grol R, Grimshaw J. From best evidence to best practice: effective implementation of change in patients' care. Lancet. 2003;362:1225-30.

43. Michie $S$, Johnston M, Francis J, Hardeman W, Eccles M. From theory to intervention: mapping theoretically derived behavioural determinants to behaviour change techniques. Appl Psychol. 2008;57:660-80.

44. French SD, Green SE, O'Connor DA, McKenzie JE, Francis JJ, Michie S. Developing theory-informed behaviour change interventions to implement evidence into practice: a systematic approach using the theoretical domains framework. Implement Sci. 2012;7:38.

45. Chaillet N, Dube E, Dugas M, Audibert F, Tourigny C, Fraser WD, Dumont A. Evidence-based strategies for implementing guidelines in obstetrics: a systematic review. Obstet Gynecol. 2006;108:1234-45.

46. Atkins L, Francis J, Islam R, O'Connor D, Patey A, Ivers N, Foy R, Duncan EM, Colquhoun $H_{\text {, Grimshaw } J M}$, et al. A guide to using the theoretical domains framework of behaviour change to investigate implementation problems. Implement Sci. 2017;12:77.

47. Damschroder $\sqcup$, Aron DC, Keith RE, Kirsh SR, Alexander JA, Lowery JC. Fostering implementation of health services research findings into practice: a consolidated framework for advancing implementation science. Implement Sci. 2009;4:50.

48. Hawkins N, Sanson-Fisher R, Shakeshaft A, et al. The multiple baseline design for evaluating population-based research. Am J Prev Med. 2007:33:162-8.

49. Sanson-Fisher R, Bonevski B, Green L, et al. Limitations of the randomised controlled trial in evaluating population-based research. Am J Prev Med. 2007:33:155

50. Hussey M, Hughes J. Design and analysis of stepped wedge cluster randomised controlled trials. Contemp Clin Trials. 2007;28:182-91.

51. Australian Institute of Health and Welfare. Australia's mothers and babies 2015 - in brief. Canberra; 2017.

52. Centre for Epidemiology and Evidence. New South Wales mothers and babies 2016. Sydney: NSW Ministry of Health; 2017. 
53. Burns E, Gray R, Smith LA. Brief screening questionnaires to identify problem drinking during pregnancy: a systematic review. Addiction. 2010;105:601-14

54. Get Healthy in Pregnancy. https://www.gethealthynsw.com.au/program/gethealthy-in-pregnancy/. Accessed 22 June 2018.

55. Michie S, Johnston M, Abraham C, Lawton R, Parker D, Walker A. Making psychological theory useful for implementing evidence based practice: a consensus approach. Qual Saf Health Care. 2005;14:26-33.

56. Cane J, O'Connor D, Michie S. Validation of the theoretical domains framework for use in behaviour change and implementation research. Implement Sci. 2012;7:37.

57. Crawford-Williams F, Steen M, Esterman A, Fielder A, Mikocka-Walus A. "If you can have one glass of wine now and then, why are you denying that to a woman with no evidence": knowledge and practices of health professionals concerning alcohol consumption during pregnancy. Women Birth. 2015;28:329-35.

58. McParlin C, Bell R, Robson S, MuirHead C, Araujo-Soares V. What helps or hinders midwives to implement physical activity guidelines for obese women? A questionnaire survey using the theoretical domains framework. Midwifery. 2016;

59. Boscart VM, Fernie GR, Lee JH, Jaglal SB. Using psychological theory to inform methods to optimize the implementation of a hand hygiene intervention. Implement Sci. 2012;7:1-12.

60. Lawton R, Heyhoe J, Louch G, Ingleson E, Glidewell L, Willis TA, McEachan RR, Foy R. Using the Theoretical Domains Framework (TDF) to understand adherence to multiple evidence-based indicators in primary care: a qualitative study. Implement Sci. 2016;11:113.

61. Proctor E, Silmere H, Raghavan R, Hovmand P, Aarons G, Bunger A, Griffey R, Hensley M. Outcomes for implementation research: conceptual distinctions, measurement challenges, and research agenda. Adm Policy Ment Health Ment Health Serv Res. 2011;38:65-76.

62. Weiner BJ, Lewis CC, Stanick C, Powell BJ, Dorsey CN, Clary AS, Boynton MH, Halko H. Psychometric assessment of three newly developed implementation outcome measures. Implement Sci. 2017;12:108.

63. McElwaine KM, Freund M, Campbell EM, Knight J, Bowman JA, Wolfenden L, McElduff P, Bartlem KM, Gillham KE, Wiggers JH. Increasing preventive care by primary care nursing and allied health clinicians: a non-randomized controlled trial. Am J Prev Med. 2014;47:424-34.

64. Wolfenden L, Yoong SL, Williams CM, Grimshaw JM, Durrheim DN, Gillham KE, Wiggers JW. Embedding researchers in health service organizations improves research translation and health service performance: the Australian Hunter New England Population Health example. J Clin Epidemiol. 2017;85:3-11.

65. Flodgren G, Parmelli E, Doumit G, Gattellari M, O’Brien MA, Grimshaw J. Local opinion leaders: effects on professional practice and health care outcomes. Cochrane Database Syst Rev. 2011;(8):CD000125.

66. Rotter T, Kinsman L, James EL, Machotta A, Gothe H, Willis J, Snow P, Kugler J. Clinical pathways: effects on professional practice, patient outcomes, length of stay and hospital costs. Cochrane Database Syst Rev. 2010;(3): CD006632.

67. Shojania KG, Jennings A, Mayhew A, Ramsay CR, Eccles MP, Grimshaw J. The effects of on-screen, point of care computer reminders on processes and outcomes of care. Cochrane Database Syst Rev. 2014;(3):CD001096.

68. Woo K, Milworm G, Dowding D. Characteristics of quality improvement champions in nursing homes: a systematic review with implications for evidence-based practice. Worldviews Evid-Based Nurs. 2017;14:440-6.

69. Welsh SM, Sherriff A, Flodgren $\mathrm{G}$. The champion for improved delivery of care to older people in long-term care settings: effects on professional practice, quality of care and resident outcomes. Cochrane Database Syst Rev. 2015;(11):CD011956

70. Reeves S, Perrier L, Goldman J, Freeth D, Zwarenstein M. Interprofessional education: effects on professional practice and healthcare outcomes (update). Cochrane Database Syst Rev. 2013;(3):CD002213.

71. Forsetlund L, Bjorndal A, Rashidian A, Jamtvedt G, O'Brien MA, Wolf F, Davis D, Odgaard-Jensen J, Oxman AD. Continuing education meetings and workshops: effects on professional practice and health care outcomes. Cochrane Database Syst Rev. 2009;(2):Cd003030.

72. Alcohol. http://www.alcohol.gov.au/. Accessed 22 June 2018.

73. Ivers N, Jamtvedt G, Flottorp S, Young JM, Odgaard-Jensen J, French SD, O'Brien MA, Johansen M, Grimshaw J, Oxman AD. Audit and feedback: effects on professional practice and healthcare outcomes. Cochrane Database Syst Rev. 2012;(3):CD000259.

74. O'Brien MA, Rogers S, Jamtvedt G, Oxman AD, Odgaard-Jensen J, Kristoffersen DT, Forsetlund L, Bainbridge D, Freemantle N, Davis D, et al. Educational outreach visits: effects on professional practice and health care outcomes. Cochrane Database Syst Rev. 2007;(4):CD000409.
Ready to submit your research? Choose BMC and benefit from:

- fast, convenient online submission

- thorough peer review by experienced researchers in your field

- rapid publication on acceptance

- support for research data, including large and complex data types

- gold Open Access which fosters wider collaboration and increased citations

- maximum visibility for your research: over $100 \mathrm{M}$ website views per year

At BMC, research is always in progress.

Learn more biomedcentral.com/submissions 